PROCEEDINGS OF THE AMERICAN MATHEMATICAL SOCIETY

Volume 124, Number 8, August 1996

\title{
ON THE SYZYGIES OF FLAG MANIFOLDS
}

\author{
LAURENT MANIVEL
}

(Communicated by Eric M. Friedlander)

\begin{abstract}
We show that on a complex flag manifold, a very ample line bundle which is a $p$-th power has property $N_{p}$ in the sense of Green and Lazarsfeld. This is a partial answer to a problem raised by Fulton.
\end{abstract}

\section{INTRODUCTION}

Let $X$ be a smooth complex projective variety, and $L$ a very ample line bundle on $X$ defining an embedding of $X$ into the projective space of hyperplanes of $\Gamma(X, L)$. Recall that $L$ has property $N_{0}$ if $X$ is embedded as a projectively normal subvariety, property $N_{1}$ if, moreover, its ideal is generated by quadrics, property $N_{2}$ if the syzygies of a minimal set of quadratic generators are linear, and property $N_{p}$ if this linearity property holds up to the syzygies of order $p-1$.

Ein and Lazarsfeld recently obtained general results about properties $N_{p}$ of adjoint bundles of very ample line bundles [E-L]. Namely, they proved that if $L$ is a very ample line bundle on some $n$-dimensional smooth projective variety $X$, and $M$ is a numerically effective line bundle, then $K_{X} \otimes L^{n+p+1} \otimes M$ has property $N_{p}$.

An outstanding problem, which was raised by Fulton ([E-L], Problem 4.5), is to understand properties $N_{p}$ of complex rational homogeneous spaces embedded by their very ample line bundles. Even for the simplest cases of Veronese embeddings of projective spaces, the answers seem to be unknown. Needless to say that, as for Plücker embeddings of grassmannians, the syzygies are extremely difficult to compute.

In this note, we try to understand the case of an ordinary flag manifold $X=G / P$, where $G=S l(V)$ is the special linear group of some complex vector space $V$ and $P$ is a parabolic subgroup. A very ample line bundle $\mathcal{L}_{\mu}$ on $X$ is defined by some character $\mu$ of $G$, restricted to $P$. Our main result will be the following:

Theorem. Suppose that the line bundle $\mathcal{L}_{\mu}$ is a p-th power of an ample bundle or, more generally, that $\mathcal{L}_{\mu}=\mathcal{L}_{\nu}^{\otimes p} \otimes \mathcal{L}_{\rho}$, where $\mathcal{L}_{\nu}$ is ample and $\mathcal{L}_{\rho}$ is generated by global sections. Then $\mathcal{L}_{\mu}$ has property $N_{p}$.

Recall that $\mathcal{L}_{\mu}$ embeds $X=G / P$ into the projective space $\mathbb{P}\left(S_{\mu} V^{*}\right)$ of hyperplanes of the so-called Schur power $S_{\mu} V$, which is the space of global sections of $\mathcal{L}_{\mu}$ on $X$, and an irreducible $G$-module of highest weight $\mu$.

Received by the editors November 28, 1994.

1991 Mathematics Subject Classification. Primary 14M15; Secondary 13D02, 14F17.

Key words and phrases. Syzygies, flag manifolds, Schur functors, Bott's theorem.

(C)1996 American Mathematical Society 
Similar results were obtained by Kempf for abelian varieties [Ke]. Note that the general results of Ein and Lazarsfeld would only imply property $N_{p}$ when $\mathcal{L}_{\mu}$ is a $p+a(X)$-th power of an ample bundle, for some large constant $a(X)$ close to the dimension of $X$. For example, when $X$ is a grassmannian of $r$-planes of the $d$-dimensional vector space $V, a(X)=(r-1)(d-r-1)$ is the Castelnuovo-Mumford regularity of the very ample generator of the Picard group of $X$.

Our approach is based on the cohomological criterion of Green and Lazarsfeld for property $N_{p}$ to hold, which involves the kernel of the evaluation map of the given line bundle. In the context of flag manifolds, we construct this evaluation map through repeated applications of Schur functors, and we deduce from that interpretation suitable resolutions of its kernel. We are then reduced to proving certain vanishing theorems for homogeneous vector bundles, which we deduce from Bott's theorem.

\section{Preliminaries}

2.1. Syzygies of projective varieties. Let $X$ be a smooth $n$-dimensional projective variety, and $L$ a very ample line bundle on $X$, defining an embedding

$$
\varphi_{L}: X \hookrightarrow \mathbb{P}\left(V^{*}\right),
$$

where $V=\Gamma(X, L)$ is the space of global sections of $L$. The algebra

$$
R=\bigoplus_{k} \Gamma\left(X, L^{\otimes k}\right)
$$

is a graded module over the symmetric algebra $S$ of $V$. Consider a minimal resolution of $R$ by free $S$-modules:

$$
\cdots \rightarrow \bigoplus_{i} S\left(-a_{i, j}\right) \rightarrow \cdots \rightarrow \bigoplus_{i} S\left(-a_{i, 1}\right) \rightarrow \bigoplus_{i} S\left(-a_{i, 0}\right) \rightarrow R \rightarrow 0 .
$$

The integers $a_{i, j}$ are called graded Betti numbers of $X$ embedded by $L$. Moreover, $L$ is said to have property $N_{0}$ if this embedding is projectively normal, i.e., $\bigoplus_{i} S\left(-a_{i, 0}\right)=S$, and to have property $N_{p}, p \geq 1$, if moreover $a_{i, j}=j+1$ for $1 \leq j \leq p$. Otherwise said, $X$ has property $N_{1}$ when its homogeneous ideal is generated by quadrics, property $N_{2}$ if, moreover, the syzygies between a minimal set of such quadratic generators are linear, and so on.

Property $N_{p}$ is equivalent to a simple cohomology condition ([E-L], Lemma 1.6). Indeed, denote by $M$ the kernel of the evaluation map of $L$ :

$$
0 \rightarrow M \rightarrow V \otimes \mathcal{O}_{X} \rightarrow L \rightarrow 0 .
$$

Suppose that $H^{1}\left(X, L^{\otimes l}\right)=0$ for $l>0$. Then $L$ has property $N_{p}$ if and only if $H^{1}\left(X, \wedge^{i} M \otimes L^{\otimes l}\right)=0$ for $l>0$ and $1 \leq i \leq p+1$. This is the cohomological criterion we want to apply on flag manifolds.

2.2. Schur complexes. Let $V$ be a complex vector space of dimension $d$, and $\mu$ a nonincreasing sequence of $d$ relative integers. Such a sequence identifies with a dominant weight of the linear group $G l(V)$, and therefore defines an irreducible rational $G l(V)$-module denoted by $S_{\mu} V$, and called the Schur power of $V$ of exponent $\mu$. This rational module is polynomial precisely when the components of $\mu$ are nonnegative, in which case we consider $\mu$ as a partition, and denote by $|\mu|$ the sum of its parts. 
Suppose given an exact sequence of vector spaces $0 \rightarrow A \rightarrow B \rightarrow C \rightarrow 0$. It is well known that any symmetric or wedge power of $C$ can then be resolved by tensor products of wedge and symmetric powers of $A$ and $B$. More generally, we can obtain a resolution of any Schur power of $C$ ([La], Lemma 1.9.1).

Proposition. Let $\mu$ be a partition. There is a complex $\mathcal{C}_{\mu}^{\bullet} \rightarrow S_{\mu} C \rightarrow 0$, whose $j$-th term is

$$
\mathcal{C}_{\mu}^{j}=\bigoplus_{|\nu|=j, \rho} c_{\nu, \rho}^{\mu} S_{\nu^{*}} A \otimes S_{\rho} B
$$

where $\nu^{*}$ is the conjugate partition of $\nu$, whose $j$-part is the number of parts of $\nu$ greater or equal to $j$, and $c_{\nu, \rho}^{\mu}$ is a Littlewood-Richardson coefficient, that is, the multiplicity of $S_{\mu} D$ in the tensor product $S_{\nu} D \otimes S_{\rho} D$ for some vector space $D$ of large enough dimension. In particular, the length of this complex is $|\mu|$, and

$$
\mathcal{C}_{\mu}^{0}=S_{\mu} B, \quad \mathcal{C}_{\mu}^{|\mu|}=S_{\mu^{*}} A .
$$

Proof. Tensor the given exact sequence by some auxiliary vector space $D$, write the usual resolutions of symmetric powers of $C$, for example, use the Cauchy formulas

$$
S^{m}(V \otimes W)=\bigoplus_{|\mu|=m} S_{\mu} V \otimes S_{\mu} W, \quad \wedge^{m}(V \otimes W)=\bigoplus_{|\mu|=m} S_{\mu^{*}} V \otimes S_{\mu} W
$$

to decompose wedge and symmetric powers of tensor products, and conclude by factoring out each Schur power of $D$ with the help of Schur's lemma for $G l(D)$.

We will use repeatedly the following standard lemma to deduce vanishing properties of cohomology groups from suitable resolutions or filtrations:

Lemma. Let $E$ be some vector bundle on $X$.

- Suppose that a resolution $\mathcal{E}^{\bullet} \rightarrow E \rightarrow 0$ is given, and that $H^{q+j}\left(X, \mathcal{E}^{j}\right)=0$ for all $j \geq 0$. Then $H^{q}(X, E)=0$.

- Suppose that a filtration of $E$ is given, and denote by gr $E$ the direct sum of the successive quotients of that filtration. If $H^{q}(X$, gr $E)=0$ for some $q \geq 0$, then $H^{q}(X, E)=0$.

\section{Borel-WeIL-BotT THEORY ON FLAG MANIFOLDS}

3.1. Preliminaries. Let $G=G l(V)$ be a complex linear group, $T$ a maximal torus, $B$ a Borel subgroup containing $T$, and $P$ a parabolic subgroup containing $B$. Denote the characters of $T$ by $\varepsilon_{1}, \ldots, \varepsilon_{d}$, choose the $\varepsilon_{i}-\varepsilon_{j}$ for $i<j$ as a set of positive roots, and denote by $\alpha_{i}=\varepsilon_{i}-\varepsilon_{i+1}$ the corresponding simple roots. Let the $\omega_{i}=\varepsilon_{1}+\cdots+\varepsilon_{i}, 1 \leq i \leq d$, be the fundamental weights. We suppose that $B$ is generated by the negative roots, and that $P$ is generated by $B$ and a set of positive roots $\left\{\alpha_{i}, i \in I\right\}$.

The homogeneous space $X=G / P$ can then be identified as the manifold of flags $V$, consisting of subspaces the codimensions of which are given by $I$. On $X$ is defined a tautological flag of vector bundles

$$
0=T_{n+1} \subset T_{n} \subset \cdots \subset T_{1} \subset T_{0}=V,
$$

where we still denote by $V$ the trivial bundle of fiber $V$. We denote by $Q_{i}=T_{i} / T_{i+1}$ the successive quotients of the tautological flag, and their ranks by $r_{i}, 0 \leq i \leq n$. 
3.2. Bott's theorem on flag varieties. Let us call a weight $\lambda P$-dominant if $\left(\lambda, \alpha_{i}\right) \geq 0$ for $i \notin I$. Among $P$-dominant weights are the characters of $P$, for which $\left(\lambda, \alpha_{i}\right)=0$ if $i \notin I$. Each $P$-dominant weight $\lambda$ defines an irreducible representation of $P$, hence an irreducible homogeneous vector bundle $\mathcal{E}_{\lambda}$ on $X$. When $\lambda$ is a character, we obtain a line bundle which we rather denote by $\mathcal{L}_{\lambda}$. Note that the Levi factor of $P$ is isomorphic to $G l_{r_{0}} \times \cdots \times G l_{r_{n}}$, and that there is an isomorphism

$$
\mathcal{E}_{\lambda} \simeq S_{\lambda_{0}} Q_{0} \otimes \cdots \otimes S_{\lambda_{n}} Q_{n}
$$

if we write $\lambda=\left(\lambda_{0}, \ldots, \lambda_{n}\right)$ with $\lambda_{i}$ a nonincreasing sequence of integers of length $r_{i}$ : such a sequence defines a dominant weight of $G l_{r_{i}}$, hence an irreducible representation that we denote, as usual, as a Schur power. When $\lambda_{i}$ has nonnegative components, we will consider it as a partition.

Let $\delta=\omega_{1}+\cdots+\omega_{d}$ be the sum of the fundamental weights. Recall that a weight $\lambda$ is said to be regular if $(\lambda, \alpha) \neq 0$ for every positive root $\alpha$, and that its index is then defined as the number of positive roots $\alpha$ for which $(\lambda, \alpha)<0$. We will need the following partial version of Bott's theorem [De]:

Theorem. Let $\lambda$ be a $P$-dominant weight. Then $H^{q}\left(X, \mathcal{E}_{\lambda}\right) \neq 0$ if and only if $\lambda+\delta$ is regular of index $q$.

3.3. Vanishing theorems. We shall derive from Bott's theorem several vanishing properties of the cohomology groups of homogeneous vector bundles on flag manifolds.

Proposition. Suppose that $\mathcal{L}_{\mu}$ is a very ample line bundle on $X$ that can be written as $\mathcal{L}_{\nu}^{\otimes l} \otimes \mathcal{L}_{\rho}$, for $\mathcal{L}_{\nu}$ very ample, $l>0$ and $\mathcal{L}_{\rho}$ globally generated. Let $\lambda_{0}, \ldots, \lambda_{n}$ be partitions, and $\lambda=\left(\lambda_{0}, \ldots, \lambda_{n}\right)$. Suppose that $|\lambda| \leq q+l$ for some $q>0$. Then

$$
H^{q}\left(X, \mathcal{E}_{\lambda} \otimes \mathcal{L}_{\mu}\right)=0
$$

Proof. We have $\mathcal{E}_{\lambda}=S_{\lambda_{0}} Q_{0} \otimes \cdots \otimes S_{\lambda_{n}} Q_{n}$ and $\mathcal{L}_{\mu}=\left(\operatorname{det} Q_{0}\right)^{l_{0}} \otimes \cdots \otimes\left(\operatorname{det} Q_{n}\right)^{l_{n}}$, and our hypothesis on $\mathcal{L}_{\mu}$ is equivalent to the inequalities $l_{i}-l_{i+1} \geq l$. Suppose that the preceding cohomology group is nonzero. Following Bott's theorem, this means that $\lambda+\mu+\delta$ is regular of positive index $q$. This weight decomposes into blocks $\left(\rho_{0}, \ldots, \rho_{n}\right)$, with

$$
\rho_{i}=\left(\lambda_{i, 1}+l_{i}+R_{i}, \ldots, \lambda_{i, j}+l_{i}+R_{i}+1-j, \ldots, \lambda_{i, r_{i}}+l_{i}+R_{i+1}+1\right),
$$

where $R_{i}=r_{i}+\cdots+r_{n}$. Suppose that the $j$-th component of $\rho_{i}$ gives rise to $q_{i, j}$ inversions with components of $\lambda+\mu+\delta$ sitting on its right. Since $\rho_{i}$ is decreasing, these components must be in different blocks. If $q_{i, j}>0$, consider among these components the one on the extreme left: it is the $j^{\prime}$-th component of some $\rho_{i^{\prime}}$ for $i^{\prime}<i$, and the corresponding inversion amounts to the inequality

$$
\lambda_{i, j}+l_{i}+R_{i}+1-j>\lambda_{i^{\prime}, j^{\prime}}+l_{i^{\prime}}+R_{i^{\prime}}+1-j^{\prime} .
$$

But $\left(R_{i}+1-j\right)-\left(R_{i^{\prime}}+1-j^{\prime}\right) \geq q_{i, j}$ by hypothesis, and $\lambda_{i^{\prime}, j^{\prime}} \geq 0$, hence

$$
\lambda_{i, j}>l_{i^{\prime}}-l_{i}+q_{i, j} \geq l+q_{i, j} .
$$

Summing over the nonempty set of those $\lambda_{i, j}$ which are responsible for the inversions 
of $\lambda+\mu+\delta$, we finally get

$$
|\lambda| \geq \sum_{i, j} \lambda_{i, j} \geq \sum_{i, j}\left(q_{i, j}+l+1\right) \geq q+l+1 .
$$

The proposition is proved.

Thanks to Schur complexes, we can derive from the previous proposition other vanishing theorems, now for nonirreducible homogeneous vector bundles. If $\rho_{1}, \ldots$, $\rho_{n}$ are partitions, let

$$
\mathcal{F}_{\rho}=S_{\rho_{1}}\left(V / T_{1}\right) \otimes \cdots \otimes S_{\rho_{n}}\left(V / T_{n}\right)
$$

Corollary. With the same hypothesis as in the previous proposition, suppose that $|\lambda| \leq q+l$ for some $q>0$. Then

$$
H^{q}\left(X, \mathcal{E}_{\lambda} \otimes \mathcal{L}_{\mu} \otimes \mathcal{F}_{\rho}\right)=0
$$

Proof. We proceed by backward induction on the smallest integer $m$ such that $\rho_{i}=0$ for $i<m$. If $m=n+1$, we are reduced to the previous proposition. If $m \leq n$, consider the short exact sequence $0 \rightarrow Q_{m} \rightarrow V / T_{m+1} \rightarrow V / T_{m} \rightarrow 0$, and the associated Schur complex, $\mathcal{S}_{\rho_{m}}^{\bullet} \rightarrow S_{\rho_{m}}\left(V / T_{m}\right) \rightarrow 0$, with

$$
\mathcal{S}_{\rho_{m}}^{j}=\bigoplus_{|\alpha|=j, \beta} c_{\alpha, \beta^{*}}^{\rho_{m}} S_{\alpha} Q_{m} \otimes S_{\beta}\left(V / T_{m+1}\right) .
$$

To prove that $H^{q}\left(X, \mathcal{E}_{\lambda} \otimes \mathcal{L}_{\mu} \otimes \mathcal{F}_{\rho_{m}, \ldots, \rho_{n}}\right)=0$ for $q>0$ and $|\lambda| \leq q+l$, it is enough to verify that

$$
H^{q+j}\left(X, \mathcal{E}_{\lambda} \otimes \mathcal{L}_{\mu} \otimes \mathcal{S}_{\rho_{m}}^{j} \otimes \mathcal{F}_{\rho_{m+1}, \ldots, \rho_{n}}\right)=0
$$

for $j \geq 0$. But this bundle decomposes into a direct sum of vector bundles of the form

$$
\mathcal{E}_{\nu} \otimes \mathcal{L}_{\mu} \otimes \mathcal{F}_{\sigma_{m+1}, \rho_{m+2}, \ldots, \rho_{n}}
$$

with $|\nu|=|\lambda|+j$, so that $|\nu| \leq q+j+l$, and we are done by induction.

\section{EMBEDDings OF FLAG MANIFOLDS}

4.1. The main theorem. Suppose that the line bundle $\mathcal{L}_{\mu}$ on the flag manifold $X=G / P$ can be written as $\mathcal{L}_{\nu}^{\otimes l} \otimes \mathcal{L}_{\rho}$, with $\mathcal{L}_{\nu}$ ample, $l>0$ and $\mathcal{L}_{\rho}$ globally generated. Then $\mathcal{L}_{\mu}$ is very ample and defines an equivariant embedding of $X$ into the projective space of hyperplanes of $S_{\mu} V$. Our purpose is to prove the following

Theorem. The embedding $X=G / P \hookrightarrow \mathbb{P}\left(S_{\mu} V^{*}\right)$ defined by the line bundle $\mathcal{L}_{\mu}$ has property $N_{l}$.

Let $M_{\mu}$ be the kernel of the evaluation map $e_{\mu}: S_{\mu} V \rightarrow \mathcal{L}_{\mu}$. Following Green's cohomological criterion, we have to prove that

$$
H^{1}\left(X, \wedge^{m} M_{\mu} \otimes \mathcal{L}_{\mu}^{\otimes p}\right)=0 \text { for } p>0,1 \leq m \leq l+1
$$

Indeed, the condition $H^{1}\left(X, \mathcal{L}_{\mu}^{\otimes p}\right)=0$ for $p>0$ is automatically verified on flag manifolds, as follows from Bott's theorem, or more simply from Kodaira vanishing.

Our strategy will be very simple. We will first interpret the evaluation map $e_{\mu}$ as a composition of morphisms obtained by applying Schur functors to simple quotient morphisms. We will deduce from that interpretation a filtration of the kernel $M_{\mu}$, with quotients that have resolutions by Schur complexes. The previous vanishing 
will finally, again via Schur complexes, be a consequence of the vanishing theorems we proved in the previous section.

4.2. The kernel of the evaluation map. Let us write $\mu=\sum_{i=0}^{n} m_{i}\left(1^{s_{i}}\right)$, where $s_{i}=r_{0}+\cdots+r_{i}$, and $\left(1^{t}\right)$ is the sequence whose first $t$ components are equal to one, the remaining ones being zeros. Our hypothesis on $\mu$ is that $m_{i} \geq l$ for $0 \leq i \leq n-1$, and we may suppose that $m_{n}=0$. Truncating $\mu$, we define partitions that can also be seen as characters of $P$,

$$
\mu^{<j}=\sum_{i=0}^{j-1} m_{i}\left(1^{s_{i}}\right) \quad \text { and } \quad \mu^{\geq j}=\mu-\mu^{<j},
$$

so that $\mu=\mu^{<n} \supset \cdots \supset \mu^{<1} \supset \mu^{<0}=0$. We have the following description of the evaluation map:

Lemma. The evaluation map $e_{\mu}$ is the composition of the maps

$$
e_{\mu}^{j}: S_{\mu<j}\left(V / T_{j+1}\right) \otimes \mathcal{L}_{\mu \geq j} \rightarrow S_{\mu<j-1}\left(V / T_{j}\right) \otimes \mathcal{L}_{\mu \geq j-1}
$$

for $1 \leq j \leq n$, the morphism $e_{\mu}^{j}$ being the twist by $\mathcal{L}_{\mu \geq j}$ of the Schur type morphism

$$
f_{\mu}^{j}: S_{\mu<j}\left(V / T_{j+1}\right) \rightarrow S_{\mu<j}\left(V / T_{j}\right)=S_{\mu<j-1}\left(V / T_{j}\right) \otimes\left(\operatorname{det} V / T_{j}\right)^{m_{j-1}}
$$

induced by the projection $V / T_{j+1} \rightarrow V / T_{j}$.

This follows, for example, from the fact that there is, up to scalars, only one $G l(V)$-equivariant map from $S_{\mu} V$ to $\mathcal{L}_{\mu}$. When $X$ is a grassmannian with quotient bundle $Q$ of rank $r$, and $\mu=l\left(1^{r}\right)$, then $S_{\mu} Q=(\operatorname{det} Q)^{\otimes l}$ and the evaluation map $S_{\mu} V \rightarrow(\operatorname{det} Q)^{\otimes l}$ is simply obtained by applying $S_{\mu}$ to the quotient map $V \rightarrow Q$. The previous lemma extends this interpretation to any flag manifold.

The maps $e_{\mu}^{j}$ being surjective, we get the following

Corollary. Let $N_{j}=\operatorname{Ker} e_{\mu}^{j}$ and $K_{j}=\operatorname{Ker}\left(e_{\mu}^{j} \circ \cdots \circ e_{\mu}^{n}\right)$. Then $M_{\mu}=K_{1}$ and there are short exact sequences

$$
0 \rightarrow K_{j+1} \rightarrow K_{j} \rightarrow N_{j} \rightarrow 0 .
$$

In particular, $M_{\mu}$ has a filtration with graded quotient bundle

$$
\operatorname{gr} M_{\mu}=N_{1} \oplus \cdots \oplus N_{n} .
$$

As a consequence, note that each tensor power $M_{\mu}^{\otimes m}$ has an induced filtration with graded quotient bundle

$$
\operatorname{gr} M_{\mu}^{\otimes m}=\bigoplus_{1 \leq i_{1}, \ldots, i_{m} \leq n} N_{i_{1}} \otimes \cdots \otimes N_{i_{m}} .
$$

4.3. Proof of the theorem. To prove that $H^{1}\left(X, \wedge^{m} M_{\mu} \otimes \mathcal{L}_{\mu}^{\otimes p}\right)=0$, it is enough to show that $H^{1}\left(X, M_{\mu}^{\otimes m} \otimes \mathcal{L}_{\mu}^{\otimes p}\right)=0$. Thanks to the previous corollary, it will suffice to verify that

$$
H^{1}\left(X, N_{i_{1}} \otimes \cdots \otimes N_{i_{m}} \otimes \mathcal{L}_{\mu}^{\otimes p}\right)=0
$$

for every sequence $i_{1}, \ldots, i_{m}$. Recall that $N_{i}$ is the kernel of a twisted Schur type morphism, and can therefore be resolved by a truncated Schur complex. Namely, we have complexes $\mathcal{N}_{i}^{\bullet} \rightarrow N_{i} \rightarrow 0$, with

$$
\mathcal{N}_{i}^{j}=\bigoplus_{|\nu|=j+1, \rho} c_{\nu, \rho}^{\mu^{<i}} S_{\nu^{*}} Q_{i} \otimes S_{\rho}\left(V / T_{i+1}\right) \otimes \mathcal{L}_{\mu \geq i} .
$$


To prove the preceding vanishing, we just need to show that

$$
H^{q+1}\left(X, \mathcal{N}_{i_{1}}^{j_{1}} \otimes \cdots \otimes \mathcal{N}_{i_{m}}^{j_{m}} \otimes \mathcal{L}_{\mu}^{\otimes p}\right)=0
$$

for $j_{1}+\cdots+j_{m}=q$. But this bundle decomposes into a direct sum of $S_{\nu_{1}^{*}} Q_{i_{1}} \otimes S_{\rho_{1}}\left(V / T_{i_{1}+1}\right) \otimes \mathcal{L}_{\mu \geq i_{1}} \otimes \cdots \otimes S_{\nu_{m}^{*}} Q_{i_{m}} \otimes S_{\rho_{m}}\left(V / T_{i_{m}+1}\right) \otimes \mathcal{L}_{\mu \geq i_{m}} \otimes \mathcal{L}_{\mu}^{\otimes p}$, with $\left|\nu_{1}\right|+\cdots+\left|\nu_{m}\right|=j_{1}+\cdots+j_{m}+m=q+m$, and $\mathcal{L}_{\mu \geq i_{1}} \otimes \cdots \otimes \mathcal{L}_{\mu \geq i_{m}} \otimes \mathcal{L}_{\mu}^{\otimes p}$ can still be written as $\mathcal{L}_{\nu}^{\otimes l} \otimes \mathcal{L}_{\sigma}$, with $\mathcal{L}_{\sigma}=\mathcal{L}_{\mu \geq i_{1}} \otimes \cdots \otimes \mathcal{L}_{\mu \geq i_{m}} \otimes \mathcal{L}_{\mu}^{\otimes p-1}$ globally generated. We can therefore apply the Corollary from 3.3, which implies our previous vanishing condition for $\left|\nu_{1}\right|+\cdots+\left|\nu_{m}\right|=q+m \leq q+l+1$, that is, for $l \leq m+1$. Our proof is complete.

\section{REFERENCES}

[A-B-W] K. Akin, D. Buchsbaum, and J. Weyman, Schur functors and Schur complexes, Adv. in Math. 44 (1982), 237-278. MR 84c:20021

[B-E-L] A. Bertram, L. Ein, and R. Lazarsfeld, Vanishing theorems, a theorem of Severi, and the equations defining projective varieties, J. Amer. Math. Soc. 4 (1991), 587-602. MR 92g:14014

[De] M. Demazure, A very simple proof of Bott's theorem, Invent. Math. 33 (1976), 271-272. MR 54:2670

[E-L] L. Ein and R. Lazarsfeld, Syzygies and Koszul cohomology of smooth projective varieties of arbitrary dimension, Invent. Math. 111 (1993), 51-67. MR 93m:13006

[Gr] M. Green, Koszul cohomology and the geometry of projective varieties, J. Diff. Geom. 19 (1984), 125-171. MR 85e:14022

[Ke] G. Kempf, The projective coordinate ring of abelian varieties, Algebraic Analysis, Geometry and Number Theory (J. I. Igusa, ed.), Johns Hopkins Press, Baltimore, 1989.

[La] A. Lascoux, Syzygies des variétés déterminantales, Adv. in Math. 30 (1978), 202-237. MR 80j: 14043

[McD] I. G. Macdonald, Symmetric functions and Hall polynomials, Clarendon Press, 1979. MR 84g:05003

[P-W] P. Pragacz and J. Weyman, Ideals generated by Pfaffians, J. Algebra 61 (1979), 189-198.

[W] H. Weyl, The classical groups, Princeton University Press, 1939. MR 1:42c

Institut Fourier, Université de Grenoble I, 38402 Saint Martin D’Hères, France

E-mail address: laurent.manivel@ujf-grenoble.fr 